\title{
РАСПРОСТРАНЕННОСТЬ АУТОИММУННОГО ГАСТРИТА У ПАЦИЕНТОВ С АУТОИММУННЫМ ТИРЕОИДИТОМ
}

\author{
Гавриленко T.E.
}

\author{
ГУ «РНПЦ радиачионной медицины и экологии человека», Гомель, Беларусь
}

Аутоиммунный тиреоидит (АИТ) встречается у 3-4\% населения Земли и в 10-15 раз чаще регистрируется у лиц женского пола. У взрослого населения случаи АИТ регистрируются чаще после беременностей, родов, абортов, в возрасте старше 35 лет, в пременопаузе и постменопаузе. Семейный анамнез АИТ имеет место в $25-30 \%$ случаев.

Распространенность аутоиммунного гастрита в структуре патологии желудка составляет 10-16\% согласно разным авторам. Аутоиммунный компонент вызывает воспалительно-дистрофические изменения в слизистом слое антрального и фундального отделов желудка. Аутоиммунный гастрит чаще всего диагностируется в хронической форме на фоне расстройств эндокринной системы, больше всего - аутоиммунного тиреоидита. Генетический аспект имеет немаловажную роль.

Выделяют 2 варианта аутоиммунного гастрита: аутоиммунный атрофический гастрит. При данной форме выделяют гастрит гипоацидный и анацидный. Такая ситуация приводит к снижению барьерной функции желудка, моторики и ухудшению процесса переваривания пищи. При таком клиническом сценарии возрастают риски развития злокачественных образований и нейроэндокринных образований ЖКТ. Основной способ диагностики-определение антител к париетальным клеткам желудка. Второй вариант - это хронический аутоиммунный гастрит. В данном случае мишенью является фактор Касла - происходит выработка специфичных антител к белку, всасывающему вит. $\mathrm{B}_{12}$ из потребляемой пищи, и, создающему защиту желудка, гастромукопротеину. Как вариант неблагоприятного прогноза- полифакторная и мегалобластная анемии, аденокарцинома. Способ диагностики - определение антител к париетальным клеткам желудка и внутреннему фактору Касла. В обоих случаях для верификации диагноза проводится эзофагогастродуоденоскопия с множественной биопсией (система стадирования OLGA/OLGIM)

С учетом специфики работы ГУ «Республиканского научно-практического центра радиационной медицины и экологии человека», а именно отделения патологии щитовидной железы и центра гастроэнтерологической канцеропревенции, нами был разработан алгоритм диагностики и тактики ведения пациентов с аутоиммунной патологией.

СУТЬ АЛГОРИтМА: при выявлении аутоиммунного тироидита пациенту рекомендуется выполнить определение уровня пепсиногенов (пепсиноген 1, 2 и соотношение 1/2), общий анализ крови, биохимическое исследование крови с обязательным определением уровня сывороточного железа и ферритина), эзофагогастродуоденоскопии (ЭГДС) с множественной биопсией. При отсутствии анемии по данным общего анализа крови и характерной морфологической картины пациент направляется на определение антител к париетальным клеткам желудка. В случае анемии - антител к париетальным клеткам желудка и внутреннему фактору Касла. Соотношение уровня пепсиногенов позволяет в достаточной мере судить об атрофических изменения в париетальных клетках желудка и своевременно диагностировать аутоиммунный атрофический гастрит. Помимо этого, определение соотношения пепсиногенов $1 \frac{2}{2}$ позволяет мониторировать динамику атрофических изменений без применения дорогостоящих, малодоступных в большинстве регионов и травматичных тестов (суточная рh-импедансометрия и множественная биопсия желудка).

За 2 года совместно с эндокринологом были обследованы 94 пациента с аутоиммунным тироидитом. В исследуемую группу вошли мужчины - 11 человек (средний возраст 46 лет) и женщины 83 человека (средний возраст 39 лет). В группе мужчин аутоиммунный атрофический гастрит был установлен у 2 пациентов (18,2\%). В группе женщин аутоиммунный атрофический гастрит выявлен у 37 человек (44,6\%) и у 2аутоиммунный гастрит без атрофии (2,4\%). Таким образом, на небольшой группе пациентов можно сделать предварительный вывод о встречаемости аутоиммунного гастрита у пациентов с АИТ. В 43,6\% случаев АИТ сочетается с аутоиммунным гастритом, при этом значительно преобладает у лицженского пола. Полученные данные будут использоваться в дальнейшем для улучшения ранней диагностики аутоиммунной патологии верхних отделов ЖКТ и разработки рекомендаций по диагностике и лечению таких групп пациентов. 